\title{
On estimation of the displacement \\ in an earthquake source and of source dimensions
}

\author{
V. KEYLIS-BOROK
}

\section{1. - INTRODUCTION.}

The study of an earthquakes mechanism is usually finished by the determination of a fault plane and the direction of displacement along it. In many aspects of geodynamics and seismology it would be very important to estimate also the dimensions of a source and chiefly - the magnitude of the displacement in it.

This problem can be reduced to the comparing of fields of statical stress before an earthquake and after it. The difference of energies of these two fields depends upon source properties in question; hence it is possible to determine dimensions of the source and the displacement in it if the form of the source and the difference of the energies be known. The energy difference can be found from observations on the seismic waves energy.

The idea of such an approach belongs to L. Knopoff. He solved the two - dimensional problem which deals with the study of very strong earthquakes with long faults. The analogical three - dimensional problem is considered in this paper; it is necessary for studying of more numerous weaker earthquakes and investigation of some contiguous problems.

\section{2. - THEORY.}

The problem can be formally stated as following.

Before an earthquake a homogeneous field of tangentional tractions exist $\tau_{x y}=p$, with corresponding displacement $u=c y, v=\frac{p}{\mu}-c x, c$ being arbitrary. We shall call this field an initial field. In the moment of the earthquake tangentional tractions vanish along the fault plane, $x^{2}+z^{2} \leqslant b^{2}, y=0$. 
A zone of crushing or intence plastic flows can appear around the fault plane. This zone must be included in the source model because the tangential tractions in it decrease or vanish; that is why we shall consider the source as an ellipsoid.

$$
\frac{x^{2}+z^{2}}{b^{2}}+\frac{y^{2}}{a^{2}}=1 \quad, \quad 0 \leqslant a \leqslant b .
$$

Now we shall examine an final field of stress which is defined by two conditions: 1 displacements and stress tend to initial ones at infinite distances; 2 the source boundary is free from stress except the hydrostatic pressure. We shall suppose the final field to appear after the earthquake with the source [1].

I neglect here plastic fiows and an inhomogeneity of the initial field which can really exist. Besides, the source boundary in the above meaning can be really not sharp.

Thus idealized problem in its main part can be reduced to this one which is already solved by Neuber in the theory of stress concentration.

I omit here ealculations which are not complicated principally and shall give the final result.

The Neuber's solution is obtained in elliptical coordinates:

$x=\operatorname{ch} u \sin v \cos w, \quad y=\operatorname{sh} u \cos v, \quad z=\operatorname{ch} u \sin v \cos w$

it can be represented by the formulas:

Normal stresses.

$$
\begin{gathered}
\sigma_{u}=\frac{p}{h^{2}} \operatorname{sh} 2 u \sin v \cos v \cos v+p \frac{C}{h^{2}}[q(T \operatorname{sh} u-2 \operatorname{chu})+ \\
+\left(c h^{2} u_{0}+1+\frac{\alpha}{2}\right) \frac{2}{c h u}-\frac{2 c h^{2} u_{0}}{c h u}-\frac{2 h^{2} u_{0}}{c h^{3} u}+ \\
\left.+\frac{2}{h^{2}}\left(\operatorname{chu}-\frac{c h^{2} u_{0}}{c h u}\right)\right] \sin v \cos v \cos w \\
\sigma_{v}=\frac{p}{h^{2}} \operatorname{sh} 2 u \sin v \cos v \cos w+p \frac{C}{h^{2}}[-q(T \operatorname{sh} 2 u-2 \operatorname{ch} u)+ \\
\left.+\left(1-\cdot h^{2} u_{\mathrm{n}}-\frac{\alpha}{2}\right) \frac{2}{c h u}-\frac{2}{h^{2}}\left(\frac{c h^{2} u_{0}}{c h u}-\operatorname{ch} u\right)\right] \sin v \cos v \cos w \\
\sigma_{w}=\frac{2 p C}{h^{2}}\left[\frac{1-\alpha}{c h u}+\frac{c h^{2} u_{0}}{c h^{3} u}\right] \sin v \cos v \cos w
\end{gathered}
$$


Tangentional stresses.

$$
\begin{aligned}
& \tau_{u v}=-\frac{p}{h^{2}}\left(\operatorname{ch}^{2} u-\frac{c h 2 u \sin ^{2} v}{h^{2}}\right) \cos w+\frac{p}{h^{2}} C\left[q\left(\operatorname{shu}-T \operatorname{sh}^{2} u\right)-\right. \\
& 2 \operatorname{sh} u-q T+\left(\operatorname{ch}^{2} u_{0}-\frac{\alpha}{2}\right)-\frac{s h u}{c n^{2} u}+\cos ^{2} v(T \operatorname{ch} 2 u-2 \operatorname{sh} u) q- \\
&\left.\quad-\cos ^{2} v\left(\operatorname{ch}^{2} u_{0}-\frac{\alpha}{2}\right) \frac{\operatorname{sh} u}{\operatorname{ch}^{2} u}+\frac{2 \operatorname{sh} u}{h^{2}}\left(c h^{2} u-\operatorname{ch}^{2} u_{0}\right)\right] \cos w \quad \\
& \tau_{m w}=\frac{p}{h} \operatorname{sh} u \sin v \sin w+\frac{p}{h} C\left[q s+\left(\operatorname{ch}^{2} u_{v}-\frac{a}{2}\right) \frac{1}{c h^{2} u}\right] \sin v \sin w
\end{aligned}
$$

$$
\tau_{u w}=-\frac{p}{h} \operatorname{ch} u \cos v \sin w+\frac{p}{h} C\left[q(\operatorname{th} u-T \operatorname{ch} u)+2 \operatorname{shu} \frac{c h^{2} u_{0}}{\operatorname{ch}^{3} u}\right.
$$

Displacements.

$$
\begin{aligned}
& \delta U_{u}=\frac{p}{2 \mu} \frac{C}{h} A_{u} \sin v \cos v \cos w \\
& \delta U_{v}=\frac{n}{2 \mu} \frac{C}{h}\left(A_{v}+A_{v} \cos ^{2} v\right) \\
& \delta U_{w}=\frac{p}{2 \mu} C A_{w} \cos v \sin w
\end{aligned}
$$

Energy-difference.

$$
\delta W=-\frac{p^{2}}{\mu} b^{3} R\left(\begin{array}{l}
a \\
b
\end{array}, a\right)
$$

$$
\begin{aligned}
& R=\left\{\left[-A_{u} \operatorname{sh} 2 u\left(\frac{1}{3}+\operatorname{sch}^{2} u_{0}\right)-A_{v} T \operatorname{sh} u_{0}+s\left(A_{v} \operatorname{sh}^{2} u_{0}-A_{v} \operatorname{ch} 2 u_{0}\right)+\right.\right. \\
& \left.\left.+\bar{A}_{v} \operatorname{ch} 2 u\left(\frac{1}{3}+s \operatorname{sh}^{2} u_{0}\right)-\frac{1}{3} A_{w} \operatorname{ch} u\right] \frac{\pi}{-2 N}+\frac{2 \pi}{3} t h u_{0}\right\}
\end{aligned}
$$

here $\sigma_{u}, \tau_{u v}$ are the stress components; $\delta U_{u}$ are the differences between the displacement in the initial and final fields; $\delta H$ is the stress energy difference (the last term $\frac{2}{3} \pi$ th $u_{0}$ in [5] represents the energy inside [1]).

$$
\alpha=\frac{\lambda+2 \mu}{\lambda+\mu} ; \quad T=\operatorname{arc} c \operatorname{tg}(\operatorname{sh} u) ; \quad h-1 \overline{\operatorname{sh}^{2} u+\cos ^{2} v}
$$




$$
\begin{aligned}
& s=T \operatorname{sh} u-1 ; \quad t h u_{0}=\frac{a}{b} ; \quad q=3 \operatorname{sh}^{2} u_{0}+1+\frac{\alpha}{2} ; \\
& C=\frac{c h^{2} u_{0}}{N\left(u_{0}\right)} ; \quad N=q(s \operatorname{sh} u+T)-2 \operatorname{sh} u_{0} . \\
& A_{u}=\left(3 \operatorname{sh}^{2} u+1\right)(2 \operatorname{ssh} u+T)-\operatorname{sh} u \frac{\operatorname{ch}^{2} u_{\mathbf{n}}}{\operatorname{ch}^{2} u}+\alpha(\operatorname{ssh} u-T) \\
& A_{v}=\left[-k+\alpha\left(s+\frac{1}{c h^{2} u}\right)\right] c h u \\
& \bar{A}_{v}=\left[2 k+\alpha\left(s-\frac{1}{c h^{2} u}\right)\right] c h u \\
& A_{w}=-k-2 \alpha s .
\end{aligned}
$$

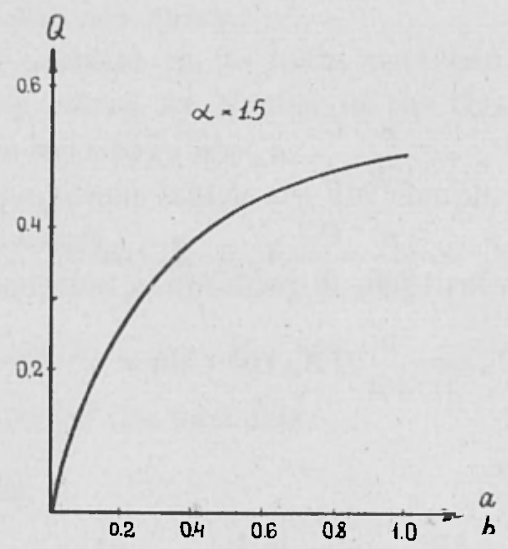

Fig. 1

The maximal tangentional stress, according to Neuber is:

$$
\tau_{\max }-\tau_{x y \mid x=y=0}-\frac{p}{Q\left(-\frac{a}{b}, \alpha\right)}, \quad Q=\frac{N s h u_{\mathrm{n}}}{\alpha}
$$

It is interesting to note, that $\tau_{\max }$ is always more than $\sim 2 p$ (fig. 1 ). The displacements change quaintly from point to point. For the measure of displacement we shall take $V$ - the maximal one on the $y$-axis, that is in the middle of the source boundary:

$$
V=\frac{p b}{\mu} \bar{\nu} ; \quad \bar{\nu}=\left.\frac{c h u}{-2 N}(k+1-2 a s)\right|_{u=u_{0}}
$$


In particular, it is interesting to consider the displacements at the source boundary $\left(u=u_{0}\right)$. If the source is thin $(a=0)$, we have:

$$
\begin{aligned}
& \delta U_{x}= \pm \frac{p}{\mu} \frac{4 \alpha}{\pi(2+\alpha)} \mid \overline{b^{2}-\varrho^{2}} \\
& \delta U_{y}=\frac{p}{\mu} \frac{\alpha-1}{2+\alpha} x \\
& \delta U_{z}=0
\end{aligned}
$$

here sings " $+"$ and " - " correspond to displacements in upper and lower sides, so that the fault does exist in our model.

It can be seen from this that in elastic case the fault plane undergoes also a rotation $\left(\delta D_{y} \neq 0\right)$ which reestablishes the statical equilibrium after the forming of a fault. It is not clear whether this rotation will take place in fact because the reestablishment of the equilibrium can be the result of imperfect elasticity. This will not change the order of quantities studied here. However the rotation will essentially distort the seismic waves, generated after the formation of a fault; therefore in constructing of the dynamic model of a fault it is necessary to allow for the plasticity near the source. (Of course, even in elastic case the above formulae can not be used directly for construction of dynamic model because they represent only its zero-frequency component).

\section{3. - INTERPRETATION.}

The following formulae can be of the main interest for the interpretation of seismic data:

$$
\begin{gathered}
S=\delta b^{2}=\left[\frac{\mu}{p^{2}} \frac{\pi W}{R_{s}\left(\frac{a}{b}, \alpha\right)}\right]^{2 / 3} \\
V=\frac{p}{\mu} \sqrt{S} L_{s}\left(\frac{a}{b}, \alpha\right)=\left(\frac{p}{\mu}\right)^{1 / 3} \overline{\delta W} L_{E} \\
p V S=\delta W \frac{R \pi}{L}
\end{gathered}
$$

where $\delta W$ is the difference of energies of the initial and final fields, $V$ is our measure of displacement, $S$ is the fault plane area and $L_{s}, R_{s}$ are plotted on fig. 2 .

$$
\left(R_{\mathrm{s}}=R \cdot \pi^{-3 / 2} ; L_{\mathrm{s}}=L \pi^{-1 / 2} L_{E}=L R^{-1 / 3}\right) .
$$


By means of these formulae one can try to determine the fault plane area $S$ and the displacement $V$ in the source if the energy of seismic waves $E$ is known. Because of energy dissipation $E$ is less than $\delta W$. However the ratio $\frac{E}{o W}$ is much more for earthquakes than for explosions and according to Byerly $E$ and $\Delta W$ are of the same order.

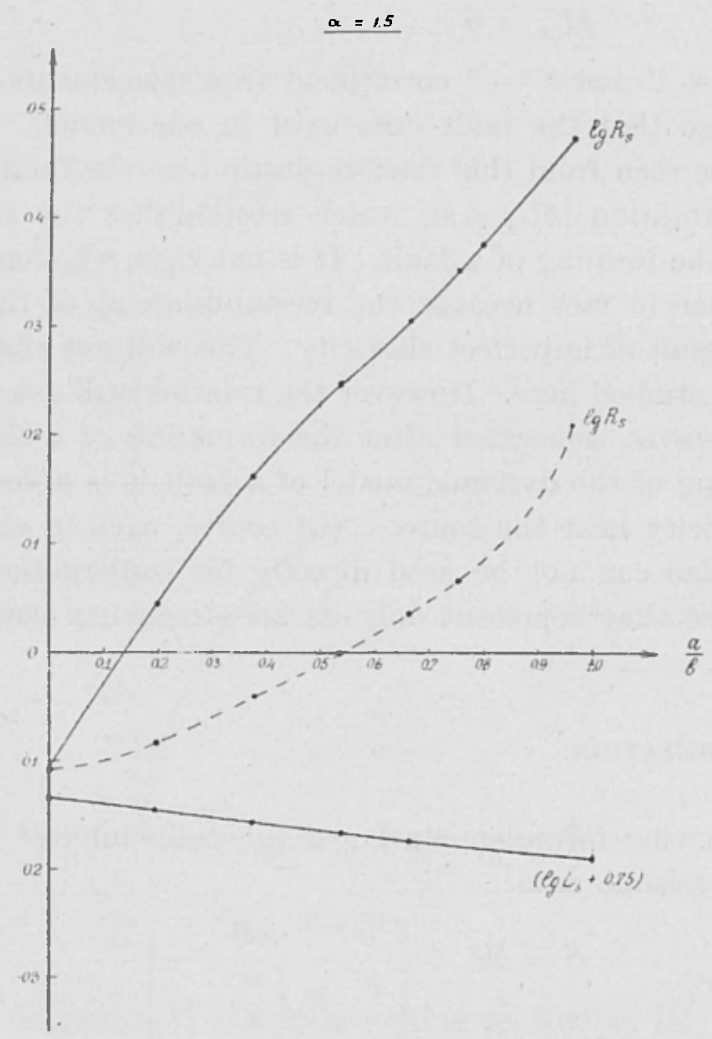

Fig. 2

It is better to use these formulae in logarithmic forn which corresponds to the accuracy of $E$ - determination.

The values of the coefficient $R_{s}, L_{s}$ are given in fig. 2 for the case $a=1.5$, which corresponds to the Poisson coefficient $v=0,25$; the variation of $\nu$ in reasonable limits $(0.23-0.27)$ may be neglected. The dashed line corresponds to the case when the energy inside the source model (ellipsoid [1]) is not included in $\delta W$ and is considered as dissipated in the plastic flow hear the source. 
The stress $p$ in the initial field is not known and this is the main difficulty in using formulas [6-8]. It may be asserted only that $p$ is less than the strengh limit as it corresponds to an average stress in a great region.

H. Benioff estimated $p$ for Kern county earthquake of 1951 from the formula $E=\frac{p^{2}}{2 \mu} H F \quad(H=$ thickness of the crust, $F$-area of aftershocks' zone); the formula is based on the assumption that the energy $E$ of seismic waves was accumulated in the zone coinciding with the zone of aftershocks. He obtained $p=2.6^{7} \mathrm{dyne} / \mathrm{cm}^{2}$. Since $E$ is known for this earthquake ( $\left.\sim 5.10^{22} \mathrm{ergs}\right) ~ p$ can be estimated using [6].

The movement took place along the fault $\sim 60 \mathrm{~km}$ long. Designating the depth of the rupture in kilometres by $H$ we obtain $S=6 H 10^{11} \mathrm{~cm}^{2}$. Then with $\mu=5.10^{11} \mathrm{cgs}$ and $a=0$ we get

$$
5 \cdot 10^{22} \simeq \frac{{ }^{n^{2}}}{5 \cdot 10^{11}}\left(H \cdot 6 \cdot 10^{11}\right)^{3 / 2} 1,1
$$

$H$ is unlikely to be greater than $35 \mathrm{~km}$. (the thickness of the crust in California) and less than $15 \mathrm{~km}$. Than $p \sim(5-9) \cdot 10^{7} \mathrm{cgs}$.

Since the source of this earthquake is greatly elongated if would be more reasonable to use formulas for two-dimensional problems. Assuming again that $H=$ from 15 to $35 \mathrm{~km}$, we obtain from the formulas of Knopoff: $p \sim(1.5-3.4) \cdot 10^{7} \mathrm{cgs}$. The obtained values of $p$ are of the same order; we shall try to use them for the study of other earthquakes.

In conclusion it should be noted that in a number of paper the volume $V$ of the region of stress accumulation was estimated from the energy of earthquakes $E$ applying the formula:

$$
E=\frac{p^{2}}{2 \mu} v .
$$

The value $v$ should not be mixed up with the volume of the model [1]: the latter may be equal to zero (with $a=0$ ); although $E$ remains finite. One can see from fig. 2 that $E$ relatively little depends upon the volume of the source and is determined mainly by the area of a fault and by the initial stress $p$.

Formulas [2], [3] can be used also for the estimating of the influence of earthquakes on the stress field around the source. In a small region around [1] the tangentional stress diminishes near the $x$-axis and becomes maximal near $z$-axis (where the screw-deformation occurs). This 
can stimulate the expansion of the fault or the formation of new faults along $z$-direction, perpendicular to the motion direction $x$. But it would be especially important to take into account non-ideal elasticity when studying the stress in the final field.

\section{4. - COMPARISON WITH OBSERVATIONS.}

Unfortunately the greater part of observational data refers to strong earthquakes with surface faults which are certainly far from the circle (fault planes are unlukly to go much deeper than the earth's crust and their horizontal length amounts to hundreds of kilometres).

For the San-Francisco earthquake of 1906 the length of a fault was $l=435 \mathrm{~km}$; assuming $S=l H$ we obtain with $H=35 \mathrm{~km}$ (the thickness of the crust in California) $S=1.5 ; 10^{14}$. Assuming $a=0$, $\mu=3.10^{11}$ we obtain from formula [7] $V \sim 3 \mathrm{~m}$. This value is of the same order that the actual one.

From formula [6] $S \sim 0.7 .10^{14} \mathrm{~cm}^{2}$ which also agrees with actual data.

It would be interesting also to compare the analogous computations with the empirical relation between $E, V$ and $S$ communicated by Don Tocher at the Toronto meeting; however he studied the strong earthquakes for which Knopoff's formulas are more valid.

In conclusion it is interesting to compare formulas [6]; [7] with the data on the frequency of occurence of earthquakes. B. Gutenberg and C. Richter showed, that with the decrease of energy of each earthquake by 100 the number of such earthquakes increases only by 10 ; therefore the part of weak earthquakes in the total amount of annually released seismic energy is very small. However with the decrease of $E$ by 100 the total area of faults will decrease (after [6]) only by $10^{1 / 3}$ and the total movement (according to [7]) will even increase by $10^{1 / 3}$. Certainly fault areas and movement values cannot be summed up in such direct way. However these estimates show that weak earthquakes can take an essential part in causing great faults or in movements along them.

(The frequency of weak earthquakes is determined for short-period ones; each of them can correspond to the movement along a part of a great fault. F. Press discovered also the long-period weak shoks, and explained them as a weak movement along a large faults as a whole).

The author is greatly thankful to prof. L. Knopoff, who acqunainted him with his paper in manuscript, and to G. Pavlova for troublesome computations. 


\section{$A B S T R A C T$}

An ellipsoid of rotation - a round fault plane surrounded by a crushing zone - is taken as a source model. The area $S$ of the fault plane and the displacement $V$ along it can be estimated using formulas:

$$
E=\frac{p^{2}}{\mu} S^{3 / 2} R_{s} ; \quad V=\frac{p}{\mu} 1 / \bar{S} L_{s}
$$

where the energy $E$ is known ( $\mu$ is shear modulus; $R_{s}, L_{s}$ coefficients given in fig. 2; $p$ mean stress before the earthquake; the value $p=3.10^{7}$ gives satisfactory results in several cases). These formulas are compared with the well-known fact that the frequency of occurrence of earthquakes is proportional to $E^{0.5}-E^{0.6}$; it is shown that the weak numerous earthquakes can take a considerable part in the formation of large faults and movement along them, though their total role in releasing seismic energy is negligibly small.

\section{RIASSUNTO}

Un elissoide di rotazione - un piano di faglia rotondo circondato da una zona di schiacciamento - è presa come modello fondamentale. $L$ 'area $S$ del piano di faglia e lo spostamento $V$ lungo questo, possono essere calcolati impiegando le formule:

$$
E=\frac{p^{2}}{\mu} \tilde{\omega}^{\prime 2} \bar{\kappa}_{s} ; \quad V=\frac{p}{\mu}-1 / \bar{S} L_{s}
$$

dove l'energia $E$ è nota ( $\mu$ è $i l$ modulo di taglio; $R_{s}, L_{\mathrm{s}} i$ coefficienti dati nella figura 2; p significa la forza prima del terremoto; il valore $p=3.10^{7}$ dà risultati soddisfacenti in diversi casi). Queste formule sono confrontate con il fatto ben noto che la frequenza con cui si verificano $i$ terremoti è proporzionale a $E^{0.5}-E^{0.0}$; si mette in rilievo che numerosi terremoti deboli possono assumere una considerevole parte nella formazione di grandi faglie e dei movimenti lungo di esse, sebbene la loro importanza totale nello sviluppo di energia sismica sia trascurabile. 


\section{REFERENCES}

Bexioff, H., Mechanism and strain characteristics of the White Wolf fault as indicated by the aftershock sequence. Kern Comnty, Cal. Earthquake of 1952, Bull. 171, Cal. Depart. of Mines, 1954.

Byeri.y, P., Report on X1 Ass. UGGI, Toronto, 1957.

K моpoff, L., Energy release in Earthquakes, "Geophys. Jorn. ", 1, 44-52, (1958).

I,URJE, A., Theree-dimentimal problems in elastostatics (in Russian). M. 1955.

Love, A., Mathematical theory of elasticity.

NeUBER, II., Kerbspannungslehre. Berlin, 1930.

Stakr, A., Slip in a cristal and rupture in a solid due to shear, Proc. "Camb. Phil. Soc. ", 24, (1928). 